\title{
VLBA DETERMINATION OF THE DISTANCE TO NEARBY STAR-FORMING REGIONS. IV. A PRELIMINARY DISTANCE TO THE PROTO-HERBIG AeBe STAR EC 95 IN THE SERPENS CORE
}

\author{
Sergio Dzib ${ }^{1}$, Laurent Loinard ${ }^{1}$, Amy J. Mioduszewski ${ }^{2}$, Andrew F. Boden ${ }^{3}$, Luis F. Rodríguez ${ }^{1}$, and Rosa M. Torres ${ }^{4}$ \\ ${ }^{1}$ Centro de Radioastronomía y Astrofísica, Universidad Nacional Autónoma de México Apartado Postal 3-72, 58090, Morelia, Michoacán, Mexico; \\ s.dzib@crya.unam.mx \\ 2 National Radio Astronomy Observatory, Domenici Science Operations Center, 1003 Lopezville Road, Socorro, NM 87801, USA \\ ${ }^{3}$ Division of Physics, Math, and Astronomy, California Institute of Technology, 1200 E California Blvd., Pasadena, CA 91125, USA \\ ${ }^{4}$ Argelander Institute for Astronomy, University of Bonn, Auf dem Hügel 71, 53121 Bonn, Germany \\ Received 2010 March 29; accepted 2010 May 22; published 2010 July 2
}

\begin{abstract}
Using the Very Long Base Array, we observed the young stellar object EC 95 in the Serpens cloud core at eight epochs from 2007 December to 2009 December. Two sources are detected in our field and are shown to form a tight binary system. The primary (EC 95a) is a $4-5 M_{\odot}$ proto-Herbig AeBe object (arguably the youngest such object known), whereas the secondary (EC 95b) is most likely a low-mass T Tauri star. Interestingly, both sources are non-thermal emitters. While T Tauri stars are expected to power a corona because they are convective while they go down the Hayashi track, intermediate-mass stars approach the main sequence on radiative tracks. Thus, they are not expected to have strong superficial magnetic fields, and should not be magnetically active. We review several mechanisms that could produce the non-thermal emission of EC 95a and argue that the observed properties of EC 95a might be most readily interpreted if it possessed a corona powered by a rotation-driven convective layer. Using our observations, we show that the trigonometric parallax of EC 95 is $\pi=2.41 \pm 0.02$ mas, corresponding to a distance of $414.9_{-4.3}^{+4.4} \mathrm{pc}$. We argue that this implies a distance to the Serpens core of $415 \pm 5 \mathrm{pc}$ and a mean distance to the Serpens cloud of $415 \pm$ $25 \mathrm{pc}$. This value is significantly larger than previous estimates $(d \sim 260 \mathrm{pc})$ based on measurements of the extinction suffered by stars in the direction of Serpens. A possible explanation for this discrepancy is that these previous observations picked out foreground dust clouds associated with the Aquila Rift system rather than Serpens itself.
\end{abstract}

Key words: astrometry - binaries: general - magnetic fields - radiation mechanisms: non-thermal - radio continuum: stars - techniques: interferometric

\section{INTRODUCTION}

An accurate knowledge of the physical properties of young stellar objects (such as their mass, age, and luminosity) is important to constrain theoretical pre-main-sequence evolutionary models. The determination of these properties, however, depends critically on the availability of accurate distances. Unfortunately, since distances to regions of star formation are often uncertain by more than $20 \%$ or $30 \%$, errors on the luminosity and age of young stars are typically about $70 \%$. Significant progress has been possible in recent years thanks to very long baseline interferometry (VLBI) observations, particularly with the Very Long Base Array (VLBA; Loinard et al. 2005, 2007, 2008; Torres et al. 2007, 2009; Menten et al. 2007; Xu et al. 2006). Owing to the very accurate astrometry delivered by such instruments, trigonometric parallaxes (and therefore distances) can be measured very precisely if multi-epoch observations spread over a few years are obtained.

VLBI instruments are only sensitive to high surface brightness emission, and can only detect objects where non-thermal processes are at work. Such non-thermal sources must, therefore, be identified in the regions of interest before their distance can be measured using multi-epoch VLBI observations. Fortunately, many young stars are magnetically active and do exhibit detectable levels of non-thermal radio emission..$^{5}$ This type of emission is typically characterized by strong variability, some

\footnotetext{
5 Theoretically, only low-mass young stars are expected to be convective and to have strong superficial magnetic fields. However, the young $6 M_{\odot}$ B4V star $\mathrm{S} 1$ in Ophiuchus (Loinard et al. 2008) is known to exhibit non-thermal radio emission, although theoretical arguments suggest it should be radiative. We will come back to this point in Section 4.2.
}

level of circular polarization, and a negative spectral index. Also, for magnetically active stars, there is a good correlation between X-ray and non-thermal radio emission (Benz \& Güdel 1994) so young stars with detectable levels of non-thermal radio emission are associated with bright $\mathrm{X}$-ray sources.

In this work, we will focus on the star-forming region associated with the Serpens molecular cloud (Strom et al. 1974; see Eiroa 1992 and Eiroa et al. 2008 for two recent reviews). More specifically, we will concentrate on the SVS 4 region (Strom et al. 1976), an infrared cluster of at least 11 pre-mainsequence sources deeply embedded within the Serpens core, and one of the densest young stellar sub-clusters known, with a stellar mass density of $\sim 10^{5} M_{\odot} \mathrm{pc}^{-3}$ (Eiroa \& Casali 1989). In the direction of SVS 4, Preibisch (1998) detected a bright $\mathrm{X}$-ray source $\left(L_{X} \sim 4 \times 10^{31} \mathrm{erg} \mathrm{s}^{-1}\right.$; Preibisch 2003a) now known to be associated with the infrared object EC 95 (Preibisch 2003a; Eiroa \& Casali 1992). Using IR spectroscopy, Preibisch (1999) showed that EC 95 is a $\sim \mathrm{K} 2$ star, and a comparison with theoretical tracks indicates that it is a very young $\left(\sim 10^{5} \mathrm{yr}\right)$, intermediate-mass star, presumably a precursor of an $\sim 4 M_{\odot}$ Herbig AeBe star. As a consequence, Preibisch (1999) argued that EC 95 is a proto-Herbig AeBe star.

Rodríguez et al. (1980) and Eiroa et al. (2005) detected EC 95 in Very Large Array (VLA) observations as an unresolved and very strong radio source (one of the strongest in the survey of Eiroa et al. 2005). This radio emission is strongly variable and has a negative spectral index, suggesting a nonthermal (gyrosynchrotron) origin (Smith et al. 1999). In order to constrain the origin of that radio emission further, Forbrich et al. (2007) obtained combined high-sensitivity VLA and HighSensitivity Array (HSA; a heterogeneous VLBI instrument 
Table 1

Measured Source Positions and Flux Densities

\begin{tabular}{|c|c|c|c|c|c|c|c|c|c|}
\hline $\begin{array}{c}\text { Mean UT Date } \\
\text { (yyyy.mm.dd) }\end{array}$ & (hh:mm) & Julian Day & $\begin{array}{c}\alpha(\mathrm{J} 2000.0) \\
18^{\mathrm{h}} 29^{\mathrm{m}}\end{array}$ & $\sigma_{\alpha}$ & $\begin{array}{l}\delta(\mathrm{J} 2000.0) \\
\quad+1^{\circ} 12^{\prime}\end{array}$ & $\sigma_{\delta}$ & $\begin{array}{c}f_{v} \pm \sigma_{f_{v}} \\
(\mathrm{mJy})\end{array}$ & $\begin{array}{c}\sigma \\
\left(\mathrm{mJy}_{\text {beam }}^{-1}\right) \\
\end{array}$ & $\begin{array}{c}T_{b} \\
\left(10^{7} \mathrm{~K}\right) \\
\end{array}$ \\
\hline \multicolumn{10}{|l|}{ EC 95a } \\
\hline 2008.11 .29 & $21: 34$ & $2,454,800.40$ & $57^{\varsigma} .8918723$ & 0.00000168 & $46 ! ' 110068$ & $0^{\prime \prime} 000078$ & $1.24 \pm 0.08$ & 0.08 & 1.71 \\
\hline 2009.02 .27 & $15: 42$ & $2,454,890.15$ & 57.8921768 & 0.00000049 & 46.106950 & 0.'000017 & $4.01 \pm 0.15$ & 0.08 & 5.55 \\
\hline 2009.12 .05 & $21: 12$ & $2,455,171.38$ & $57^{\S} .8922183$ & 0 s.00000070 & 46.095333 & $0^{\prime \prime} 000032$ & $2.77 \pm 0.16$ & 0.09 & 4.37 \\
\hline \multicolumn{10}{|l|}{ EC $95 b$} \\
\hline 2007.12 .22 & $19: 38$ & $2,454,457.32$ & 57.8909548 & 0.00000094 & $46 ! ' 108014$ & $0^{\prime \prime} 000032$ & $1.79 \pm 0.10$ & 0.05 & 2.14 \\
\hline 2008.06 .29 & 07:39 & $2,454,646.82$ & 57.8909523 & 0.00000370 & 46 .'107340 & $0^{\prime \prime} 000145$ & $0.47 \pm 0.16$ & 0.09 & 0.61 \\
\hline 2008.09.15 & $02: 33$ & $2,454,724.61$ & $57^{\$} .8908014$ & 0.00000087 & 46.105880 & $0^{\prime \prime} 000029$ & $4.51 \pm 0.23$ & 0.11 & 4.43 \\
\hline 2008.11 .29 & $21: 34$ & $2,454,800.40$ & $57^{\mathrm{S}} .8908963$ & $0^{s} .00000168$ & 46 .' 104204 & $0^{\prime \prime} 000078$ & $1.03 \pm 0.08$ & 0.08 & 1.45 \\
\hline 2009.02.27 & $15: 42$ & $2,454,890.15$ & $57^{\S} .8911287$ & $0 ؟ 00000575$ & 46 ". 103781 & 0.000148 & $0.41 \pm 0.16$ & 0.08 & 0.45 \\
\hline 2009.06 .03 & $09: 22$ & $2,454,985.89$ & $57^{\mathrm{s}} .8910820$ & 0.00000408 & 46.104144 & $0^{\prime \prime} 000096$ & $1.57 \pm 0.24$ & 0.11 & 1.96 \\
\hline 2009.08 .31 & $03: 35$ & $2,455,074.65$ & 57.8909107 & 0.00000085 & 46.103210 & $0^{\prime} .000030$ & $3.11 \pm 0.14$ & 0.07 & 4.19 \\
\hline
\end{tabular}

that includes the VLBA, the VLA, and the Effelsberg and Green Bank single-dish telescopes) observations. The source is detected with the VLA as a compact $\sim 0.5 \mathrm{mJy}$ source, but not on the long baselines of the HSA (at a $3 \sigma$ upper limit of about $50 \mu \mathrm{Jy}$ ). They argue that the emission detected at the VLA traces free-free radiation from an ionized wind that might absorb the emission from the underlying active magnetosphere. In this paper, however, we will describe new multi-epoch VLBA observations of EC 95, in which compact non-thermal emission is detected on very long baselines. We will use these data to estimate the trigonometric parallax of that source, and of the Serpens core as a whole. As discussed by Eiroa et al. (2008), the distance to Serpens has been a matter of some controversy over the years, with estimates ranging from $210 \mathrm{pc}$ to $700 \mathrm{pc}$ (see Section 5).

\section{OBSERVATIONS AND DATA CALIBRATIONS}

We present a series of eight radio continuum observations obtained at $3.6 \mathrm{~cm}(8.42 \mathrm{GHz})$, using the VLBA of the National Radio Astronomy Observatory (NRAO) between 2007 December and 2009 December. The first observation (in 2007 December) was designed as a detection experiment. Since the source was successfully detected, we then initiated a series of multi-epoch observations starting in 2008 June. The separation between successive observations in those multi-epoch data was about 3 months (Table 1).

We used the quasar J1833+0115 as the main phase calibrator; it is located at 0.79 from the target, EC 95. Also, we used the quasars $\mathrm{J} 1832+0118, \mathrm{~J} 1826+0149$, and $\mathrm{J} 1824+0124$ (located at $0.68,1.08$, and 1.30 from the target, respectively) as secondary phase calibrators (Figure 1). For astrometry, it is always desirable for the main calibrator to be strong and close to the target. In this case, we used J1833+0115 rather than J1832+0118 as a primary calibrator because the former, although slightly farther from the source, is significantly stronger than the latter. Each observation consisted of series of cycles with 2 minutes spent on source and 1 minute spent on J1833+0115. Every $\sim 30$ minutes we observed the secondary phase calibrators, spending 1 minute on each. The data were edited and calibrated using the Astronomical Image Processing System (AIPS; Greisen 2003). The basic data reduction followed the standard VLBA procedure for phase-referenced observations, including the multi-calibrator schemes. It was described in detail in Loinard et al. (2007) and Torres et al. (2007).

Even after the careful calibration described above, systematic phase errors, which adversely affect the astrometric quality of

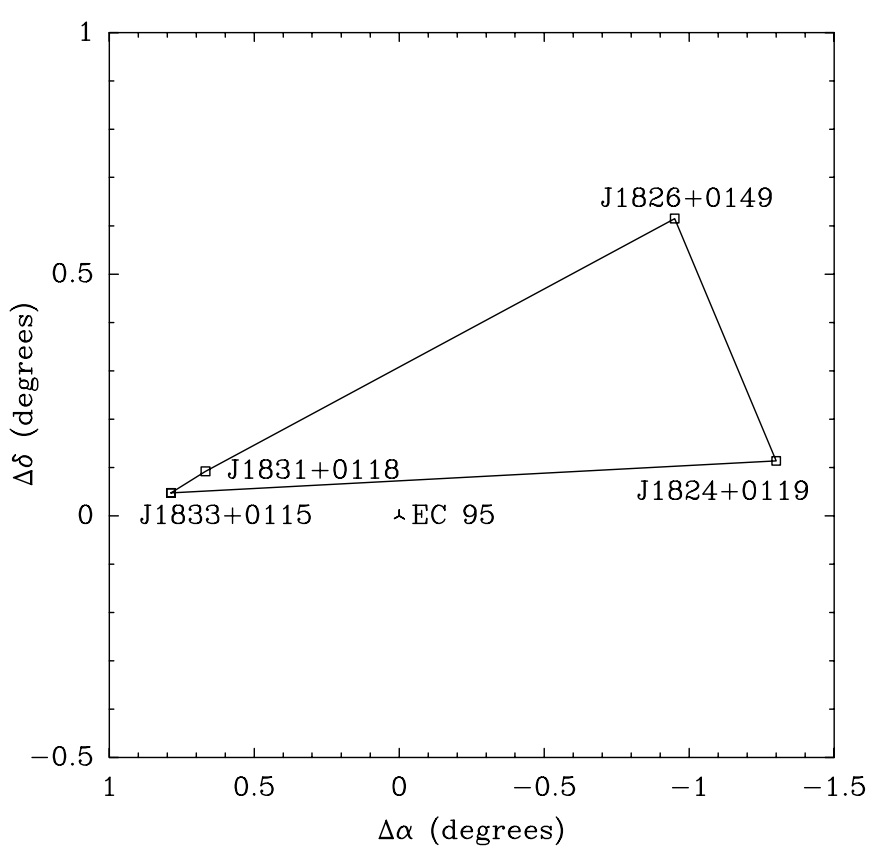

Figure 1. Relative position of the astronomical target, as well as of the main and secondary calibrators.

the data, are still present. Above $5 \mathrm{GHz}$, the largest effects come from remaining tropospheric calibration errors due to imperfections in the atmospheric model used by the VLBA correlator and from inaccuracies in the clocks used at each antenna (see Reid \& Brunthaler 2004). As a consequence of these errors, the phase correction adequate for the target is slightly different from those determined using the calibrators. To correct for these effects, one must measure the so-called group delay (i.e., the rate of phase change with frequency). This can be done by observing a dozen quasars distributed over the entire sky over a wide range of frequencies (Reid \& Brunthaler 2004). From such measurements, the variable clock delays and the tropospheric term can be obtained, and the systematic phase errors remaining after standard calibration can be minimized. We applied this strategy to the last seven epochs of our data ${ }^{6}$ using observations of multiple ICRF quasars obtained at the beginning, the middle, and the end of each observing session. Once the group delays were measured using these data, they

\footnotetext{
6 Since the first observation was designed as a detection experiment, it did not
} include the additional calibration scans required to measure the group delay. 

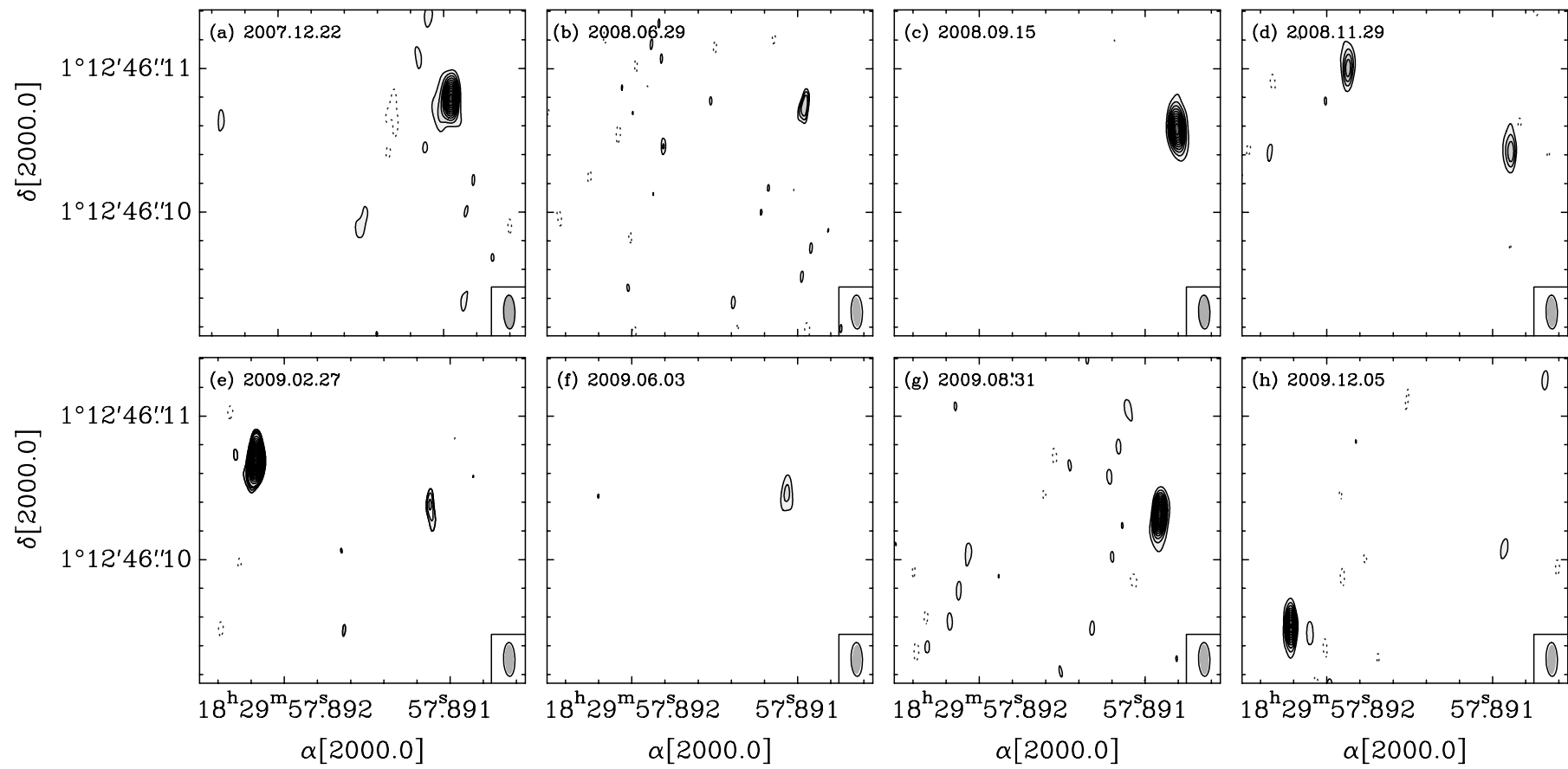

Figure 2. Images of EC 95 at all eight epochs. The synthesized beam for each observation is shown at the bottom right of each panel. The contour levels are $-3,3,9$, 12 , etc. times the noise level in each image (those noise levels are given explicitly in Table 1). Moreover, contours at $4 \sigma$ and $5 \sigma$ were added to panels (b) and (e) to bring out EC 95b, which was detected only at $5 \sigma$ at those epochs.

were applied using the dedicated task DELZN in AIPS (see Mioduszewski \& Kogan 2009).

After the data had been calibrated as described above, the visibilities were imaged with a pixel size of $50 \mu$ as using a weighting scheme intermediate between natural and uniform (ROBUST $=0$ in AIPS). The rms noise levels in the final images were $0.08-0.10 \mathrm{mJy}^{\text {beam }}{ }^{-1}$ (Table 1 ).

\section{RESULTS}

\subsection{Structure and Properties of the Emission}

In six of our eight observations, a single source was detected in the VLBA images, whereas two sources were simultaneously detected in the other two (Figure 2). As Figure 2 shows, a source is detected at $\alpha \sim 18^{\mathrm{h}} 29^{\mathrm{m}} 57^{\mathrm{s}} .891$ at seven epochs, whereas another source is detected at $\alpha \sim 18^{\mathrm{h}} 29^{\mathrm{m}} 57$. 892 only three times (panels (d), (e), and (h) of Figure 2). The location of both sources on the plane of the sky appears to change with time (Figure 2), so it is clear that neither of them is an extragalactic background object. Moreover, a comparison between panels (d) and (e) in Figure 2 clearly shows that both sources are moving on the plane of the sky in a similar fashion. Thus, they are most likely associated with one another. It would, indeed, be extremely unlikely for two Galactic radio sources separated by a mere 15 mas to be unrelated. We conclude that both radio sources detected in our VLBA observations are associated with the protostellar object EC 95. For reasons that will become apparent momentarily, we shall call the source at $\alpha \sim 18^{\mathrm{h}} 29^{\mathrm{m}} 57^{\mathrm{s}} 891$ EC 95b and at $\alpha \sim 18^{\mathrm{h}} 29^{\mathrm{m}} 57$. 892 EC 95a.

Both sources are found to be very variable (Figure 3 ). While they both can reach a flux of about $4 \mathrm{mJy}$, they sometimes remain undetected at levels below about $0.2 \mathrm{mJy}$. The brightness temperature of the emission is typically of the order of $10^{7} \mathrm{~K}$ (last column of Table 1). All these properties demonstrate that the emission is non-thermal, and strongly suggest that each radio source is associated with a flaring star. Interestingly, no circular polarization was detected in our observations - to an upper limit of about $5 \%$. This is unlike the situation in other magnetically active young stars (e.g., Loinard et al. 2007, 2008, 2009; Torres et al. 2007, Torres et al. 2009) and likely indicates that the magnetic field topology on the active stars in EC 95 is complex.

\subsection{Astrometry}

The positions of the sources in our VLBA images were determined using a two-dimensional Gaussian fitting procedure (task JMFIT in AIPS) and are given in Table 1. JMFIT provides an estimate of the position error based on the expected theoretical astrometric precision of an interferometer (Condon 1997); these errors are quoted in Columns 4 and 6 of Table 1 . To obtain the astrometric parameters from these data, we used the single value decomposition fitting scheme described by Loinard et al. (2007). The necessary barycentric coordinates of the Earth, as well as the Julian date of each observation, were calculated using the Multi-year Interactive Computer Almanac (MICA) distributed as a CD ROM by the US Naval Observatory. The reference epoch was taken at the mean of our observations: JD $2454765.98 \equiv \mathrm{J} 2008.90$.

Since EC 95a is only detected three times, it would be very hazardous to fit the observed positions with a combination of parallax and proper motions. Thus, we will concentrate here on the EC 95b component. Two fits were performed. In the first one, we assumed a linear and uniform proper motion. The best fit under this assumption is shown in the left panel of Figure 4, and yields the following astrometric elements:

$$
\begin{aligned}
\alpha_{J 2008.9} & =18^{\mathrm{h}} 29^{\mathrm{m}} 57^{\mathrm{s}} .890983 \pm 0.000009 \\
\delta_{J 2008.9} & =1^{\circ} 12^{\prime} .46^{\prime \prime} .1055 \pm 0.0001 \\
\mu_{\alpha} \cos \delta & =0.72 \pm 0.25 \mathrm{mas} \mathrm{yr}^{-1} \\
\mu_{\delta} & =-3.61 \pm 0.20 \mathrm{mas} \mathrm{yr}^{-1} \\
\pi & =2.30 \pm 0.19 \text { mas. }
\end{aligned}
$$



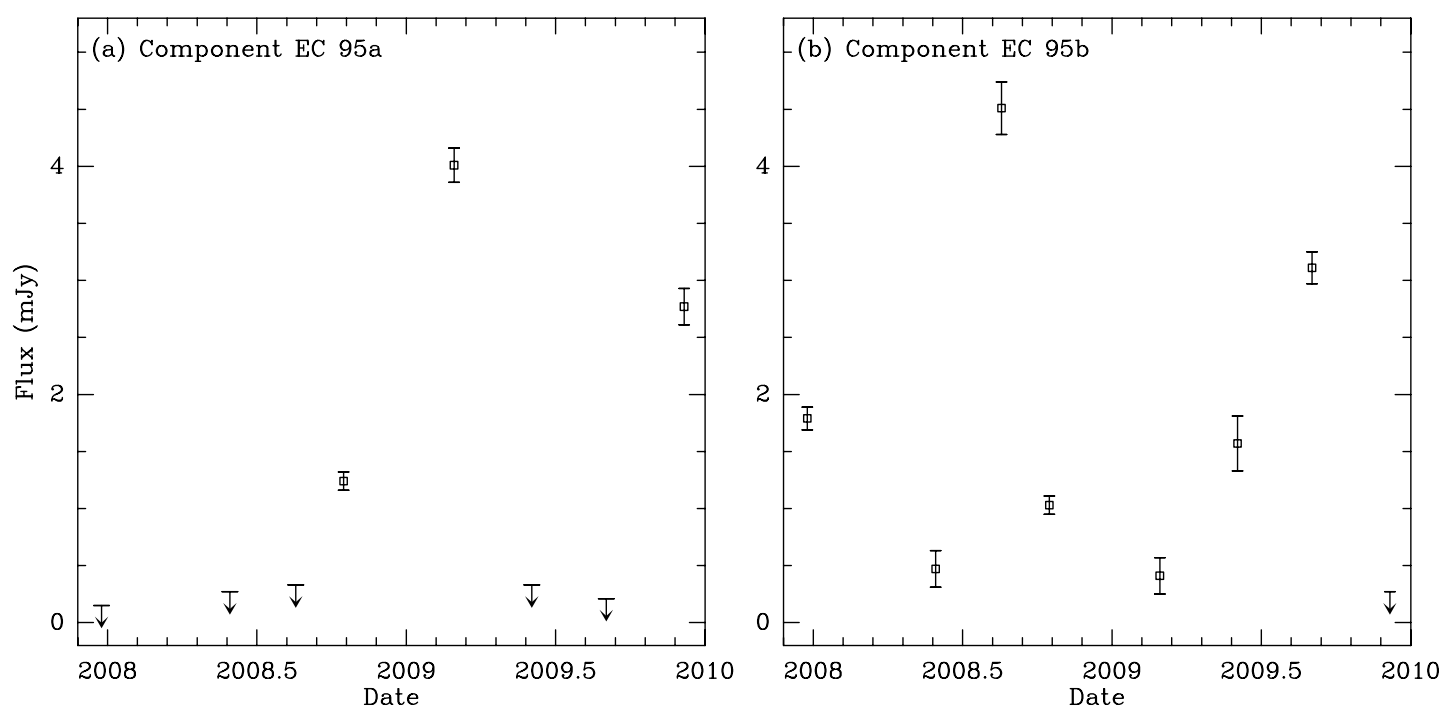

Figure 3. Time evolution of the $3.6 \mathrm{~cm}$ flux of EC 95a and EC 95b in our VLBA data. The upper limits are at $3 \sigma$.

The corresponding distance is $435.2_{-32.9}^{+38.8} \mathrm{pc}$. Note, however, that the post-fit rms are somewhat large: 0.28 and 0.21 mas in R.A. and decl., respectively. Indeed, Figure 4 shows that the observed positions often do not coincide with the positions expected from the best fit.

We saw earlier that two sources are detected in our VLBA images and that those sources are likely to trace two associated active stars. Under these circumstances, it is to be expected that they will be in gravitational interaction and that their motions will be accelerated rather than uniform. As a consequence, we performed another fit including a uniform acceleration term (similar to that included in the fit to the T Tau data; see Loinard et al. 2007). This fit is shown in the right panel of Figure 4, and yields the following parameters:

$$
\begin{aligned}
\alpha_{J 2008.9} & =18^{\mathrm{h}} 29^{\mathrm{m}} 57^{\mathrm{s}} .890966 \pm 0.000002 \\
\delta_{J 2008.9} & =1^{\circ} 12^{\prime} .46^{\prime \prime} .10532 \pm 0.00008 \\
\mu_{\alpha} \cos \delta & =0.70 \pm 0.02 \mathrm{mas} \mathrm{yr}^{-1} \\
\mu_{\delta} & =-3.64 \pm 0.10 \mathrm{mas} \mathrm{yr}^{-1} \\
a_{\alpha} \cos \delta & =1.95 \pm 0.05 \mathrm{mas} \mathrm{yr}^{-2} \\
a_{\delta} & =1.41 \pm 0.36 \mathrm{mas} \mathrm{yr}^{-2} \\
\pi & =2.41 \pm 0.02 \text { mas. }
\end{aligned}
$$

This corresponds to a distance of $414.9_{-4.3}^{+4.4} \mathrm{pc}$, consistent with the value obtained without using the acceleration terms, but nearly 10 times more accurate. The post-fit rms is 0.02 mas in R.A. and 0.10 mas in decl., significantly better than those obtained when no acceleration is included (indeed, Figure 4(b) shows that the agreement between the data and the fit is much better when acceleration is included). The reduced $\chi^{2}$ obtained in R.A. using the errors delivered by JMFIT is 0.6, suggesting that no significant systematic errors remain in our data along that axis. In decl., however, a systematic contribution of 0.127 mas has to be added quadratically to the errors given by JMFIT to obtain a reduced $\chi^{2}$ of 1 . All the errors quoted here include this systematic contribution.

\subsection{Binarity}

Adding free parameters to a fit will always improve the agreement between the fit and the data. Therefore, the improvement in our fit when acceleration terms are included does not necessarily guarantee that an accelerated trajectory provides a better description of the data. The premise for the inclusion of an acceleration term was the existence of a gravitational interaction between the two sources in our field. Accordingly, we must now verify that the acceleration vector obtained from the fit does point toward the second radio source.

To examine this issue, we subtracted the parallactic contribution from the observed positions of both EC 95a and EC 95b (Figure 5(a)). While the trajectory of EC 95b is significantly curved, that of EC 95a appears to be linear and uniform to a very good degree of precision. This confirms that EC 95a and EC $95 \mathrm{~b}$ are at the same distance since it would require an extraordinary coincidence to obtain a uniform motion for EC 95a after applying the parallax correction of EC 95b if the two sources were not at the same distance. Moreover, the acceleration vector (of EC 95b) found in our fit appears to point almost exactly toward the expected position of EC 95a at the median epoch of our observations (Figure 5(a)). This is exactly what would be expected if EC 95a and EC 95b formed a gravitationally bound binary system. Finally, since the trajectory of EC 95b is curved and accelerated, whereas that of EC 95a is linear and uniform, EC 95a must be significantly more massive than EC 95b. We conclude that EC 95a and EC 95b do constitute a binary system, where EC 95a is the primary and EC 95b is the secondary. This is, indeed, the reason why we ascribed those names to the sources in the first place.

To further characterize the system, it is useful to calculate the separation between EC 95a and EC 95b as a function of time. Since the two sources are detected simultaneously at only two epochs, this can be unambiguously done only at those two epochs. However, since the motion of EC 95a appears to be very nearly linear and uniform, we can estimate the position of the primary at the other five epochs when the secondary is detected. Those positions are shown as blue solid circles in Figure 5(a). For epochs 6 and 7, the estimation of the position of the primary only involves an interpolation. However, for the first three epochs, somewhat more uncertain extrapolations are needed. Using these estimates for the position of the primary, we calculated the separation between the two members of the system shown in Figure 5(b). It is clear from this figure that our observations only cover a fairly small fraction of the orbit and that any orbit modeling will be very uncertain. A 

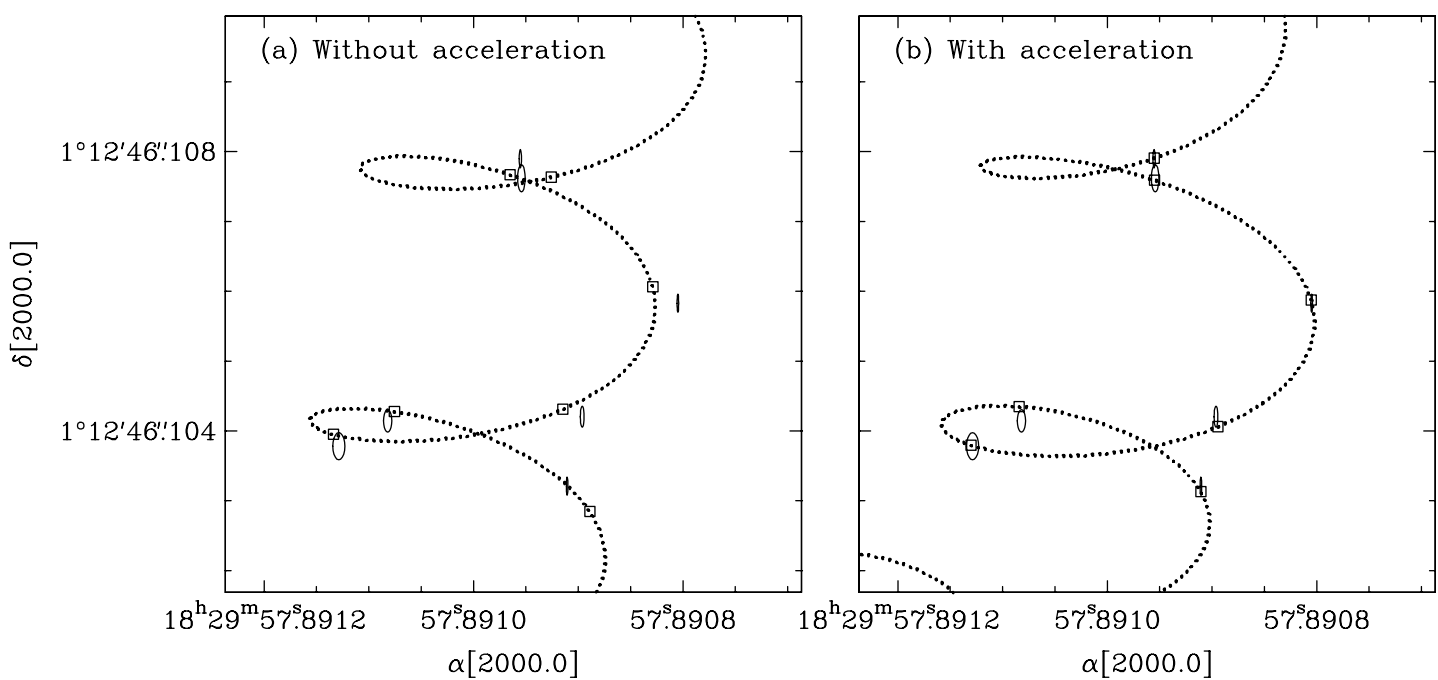

Figure 4. Observed positions of EC $95 \mathrm{~b}$ and best astrometric fits (a) without acceleration terms and (b) with acceleration terms. The squares indicate the position of the source expected from the fits at each epoch, and the ellipses show the actual observed positions. The size of the ellipse represents the error on the measurement.

poorly constrained but plausible fit (see below) is shown as a solid curve. The dotted curve corresponds to the parabolic path predicted by our uniformly accelerated fit. The fact that those two trajectories are nearly indistinguishable over the course of our observations justifies a posteriori the use of a uniformly accelerated motion in our astrometric fit.

A strict lower limit to the mass of the primary can be found from the magnitude of the acceleration vector determined earlier. If $m$ and $M$ are the masses of the secondary and the primary, respectively, Newton's law applied to the secondary yields

$$
m a=\frac{G m M}{r^{2}} \Rightarrow M=\frac{a r^{2}}{G},
$$

where $a$ is the magnitude of the acceleration (of the secondary) and $r$ is the true separation between the sources. Of course, we measure only quantities projected onto the plane of the sky, so the measured separation and acceleration are only lower limits. From the measured values $\left(a_{\min }=2.4 \mathrm{mas} \mathrm{yr}^{-2} \equiv 0.015 \mathrm{~cm} \mathrm{~s}^{-2}\right.$ and $r_{\min }=15.8$ mas $\left.\equiv 9.86 \times 10^{13} \mathrm{~cm}\right)$, we obtain a minimum mass for EC $95 \mathrm{a}$ of $1.1 M_{\odot}$. The mass goes roughly as $\cos ^{3} i$, so if the inclination were $45^{\circ}$, the mass of EC 95a would be about $3 M_{\odot}$.

To obtain an alternative mass estimate, we model the component relative astrometry data (Table 1; Figure 5(b)) with a Keplerian orbit. As these data apparently cover only a small ( $\sim 10 \%)$ fraction of the EC 95 orbit, we constrained the orbit model to be circular $(e=0)$; this assumption is not physically motivated, but the data do not presently support a more complex interpretation. The result of our modeling is shown as a solid line in Figure 5(b). The semimajor axis of this preliminary orbit is $31 \pm 9$ mas, its inclination is $i=60^{\circ} \pm 10^{\circ}$, and the orbital period is $16.5 \pm 5.0 \mathrm{yr}$. These orbit parameters imply a total mass for the system of $M=8_{-6}^{+27} M_{\odot}$. The large error on the upper limit reflects the fact that the mass goes roughly as $\cos ^{3} i$ and increases rapidly for $i$ above $45^{\circ}$. Even with this consideration, however, the orbit parameter and mass errors are likely to be underestimated because of the non-physical assumption of a circular orbit required in this very preliminary modeling.

\section{PROPERTIES OF EC 95 AND SVS 4}

\subsection{Nature of EC 95}

From near-infrared spectroscopy, Preibisch (1999) estimated a spectral type $\mathrm{K} 2 \pm 2$ for EC 95 and an extinction-corrected bolometric luminosity $L=60_{-20}^{+30} L_{\odot}$. This was obtained assuming a distance to Serpens of $310 \mathrm{pc}$; using the value of the distance obtained here increases the luminosity to $108_{-36}^{+54} L_{\odot}$. More recently, Doppmann et al. (2005) estimated the effective temperature of EC 95 to be $4400_{-57}^{+115} \mathrm{~K}$, in agreement with the spectral type proposed by Preibisch (1999), and its luminosity (calculated assuming a distance of $259 \mathrm{pc}$ ) to be $23 L_{\odot}$. Scaled to the distance determined here, this would imply a luminosity of $59 L_{\odot}$, also consistent with the determination of Preibisch (1999). The extinction determined by Preibisch (1999) is $A_{V}=36 \pm 2$; an alternative determination by Pontoppidan et al. (2004) yields $A_{V} \sim 37$.

The mass and the age of EC 95 can be estimated by placing it on an H-R diagram. Using that method, Preibisch (1999) estimated a mass of about $4 M_{\odot}$ and an age of $0.2_{-0.1}^{+0.2}$ Myr. Pontoppidan et al. (2004) proposed a similar mass $\left(3.5 M_{\odot}\right)$ and a marginally larger age $(0.4 \mathrm{Myr})$ because the shorter distance they used implies a correspondingly smaller luminosity. In any case, all these characteristics suggest that EC 95 is a very young precursor of an $\sim 4 M_{\odot}$ star. Using our new distance determination, the position of EC 95 on the H-R diagram moves up somewhat, and its position suggests a mass of about $5 M_{\odot}$ and an age of about $0.2 \mathrm{Myr}$, confirming that EC 95 is a precursor of an intermediate-mass star. In spite of their large uncertainties, the dynamical mass estimates given above are also consistent with that suggestion. Our observations further show that EC 95 is a binary system where the primary is significantly more massive than the secondary. As a consequence, we identify the proto-Herbig AeBe star with EC 95a and argue that EC 95b must be a low-mass T Tauri companion. It is clear that in such a situation, both the bolometric luminosity and the spectral type of the system will be almost entirely dominated by the more massive component. We should also point out that another young star (EC 92), located about 5" ( 2000 AU) to the north of EC 95, has been argued to be gravitationally bound to it (e.g., Haisch et al. 2002). If that were indeed the case, then 

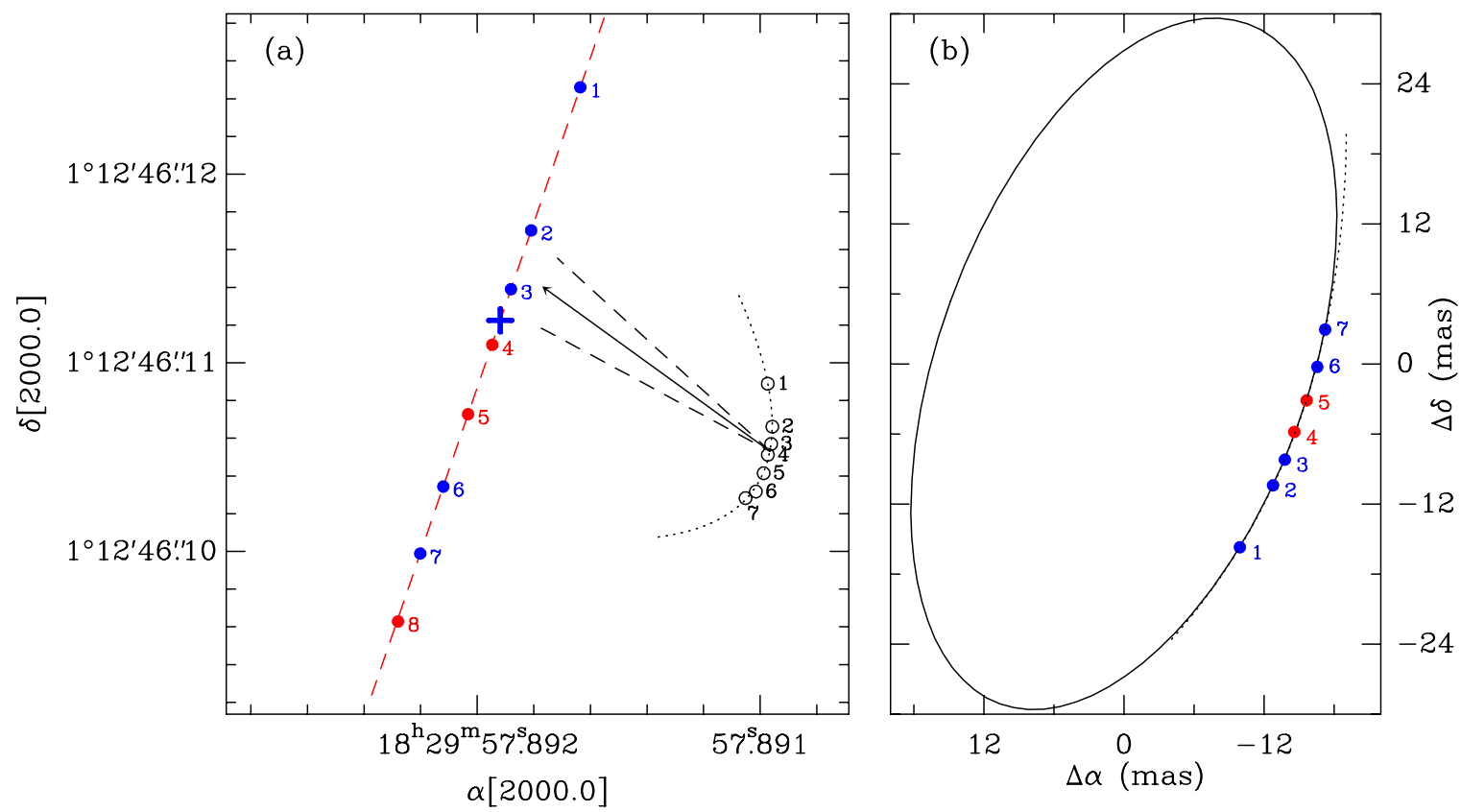

Figure 5. (a) Positions of EC 95a and EC 95b as a function of time after removing the parallactic component. The black empty circles show the positions of EC 95b, and the filled circles correspond to EC 95a. The epoch is indicated by a number on the side of each symbol. For EC 95a, the epochs for which the positions are measured are shown in red, whereas the blue symbols are for the epochs where the position was obtained using the (uniform) fit shown as a dashed line. (b) Relative position between EC 95b and EC 95a, deduced from the absolute positions shown in panel (a). The ellipse shows the best fit to the data points assuming an intrinsically circular orbit (see the text). In both panels, the dotted curve shows the best uniformly accelerated fit to the original data.

EC 92/EC 95 would constitute a rare example of a very young, intermediate-mass, hierarchical triple system.

In spite of its youth, EC 95 does not appear to contain large quantities of circumstellar material. It shows only a modest midinfrared excess (Preibisch 1999; Haisch et al. 2002; Pontoppidan et al. 2004), little veiling (Doppmann et al. 2005), and fairly weak $\mathrm{CO}$ overtone rovibrational absorption lines. This has led some authors to classify it as a Class II source (e.g., Eiroa et al. 2005), although others have favored a flat spectrum (e.g., Harvey et al. 2007) or even Class 0/I classification (Winston et al. 2007). We note, in particular, that the fairly large rotational velocity of EC $95\left(56 \mathrm{~km} \mathrm{~s}^{-1}\right.$; Doppmann et al. 2005) would be more consistent with a Class I or flat spectrum classification. It is interesting to compare these characteristics with those of the nearby, possibly associated, source EC 92 . From its location on the H-R diagram, Preibisch (1999) estimated that EC 92 is a $0.5 M_{\odot}$ star with an age of the order of $10^{5} \mathrm{yr}$. This suggests that EC 92 and EC 95 are nearly coeval (as would be natural if they belonged to a common multiple system). Unlike EC 95, however, EC 92 does exhibit a significant mid-infrared excess and has been almost unanimously classified as a flatspectrum Class I source (see e.g., Pontoppidan et al. 2004). Thus, while EC 92 and EC 95 appear to have similar ages and might be physically associated, they differ significantly in their circumstellar content. The fact that EC 95 is a tight binary system could naturally explain the relative paucity of its circumstellar content because tidal forces tend to be effective at clearing out circumstellar material. In particular, any disk existing around the members of the EC 95 system are expected to be truncated down to a radius at most about a third of the physical separation between the stars: $\sim 5$ mas ( $\equiv 2 \mathrm{AU}$ ).

\subsection{Origin of the Non-thermal Emission in EC 95}

Low-mass young stars (i.e., $\mathrm{T}$ Tauri stars) have long been known to often be magnetically active (e.g., Feigelson \&
Montmerle 1999). The accepted explanation for this activity is based on a scaled-up version of the situation with the Sun. While they move down the Hayashi track toward the main sequence, stars less massive than about $2 M_{\odot}$ are fully convective (e.g., Palla \& Stahler 1993). As a consequence, they can generate strong superficial magnetic fields $(\sim 1 \mathrm{kG})$ through the dynamo mechanism (Parker 1955). This leads to the appearance of magnetic loops anchored on the stellar surface, and extending up to a maximum height of a few stellar radii. When two loops interact, flares associated with magnetic reconnection events can occur, leading to the sudden release of large quantities of energy initially stored in the magnetic field. Part of that energy can accelerate electrons initially trapped in the loops to mildly relativistic speeds. These electrons then spiral in the strong ambient magnetic fields and generate gyrosynchrotron radiation that can be detected at radio wavelengths. The energy released during reconnection also serves to heat the stellar corona to temperatures sufficient to generate detectable thermal bremsstrahlung X-ray emission.

Stars more massive than about $2-3 M_{\odot}$ are expected to move toward the main sequence on radiative tracks and are not anticipated to have strong superficial fields. Such stars are, therefore, not expected to be magnetically active. X-ray emission and non-thermal radio emission have been detected from massive O and WR stars (e.g., Berghöfer et al. 1997; Pittard \& Dougherty 2006), but are believed to be the result of shocks in winds and wind interactions. For intermediatemass stars (spectral type A and late B), however, the situation appears somewhat more uncertain. Those stars should not be magnetically active and have no strong winds. However, a small fraction of them (perhaps about 5\%; Montmerle et al. 2005) do show evidence of strong magnetic activity (Stelzer et al. 2005, Stelzer et al. 2006; Wade et al. 2009; Hubrig et al. 2009). Several explanations have been put forward, but arguably the most plausible one is that the magnetic activity is 
in fact associated with a low-mass companion rather than with the intermediate mass primary itself (e.g., Stelzer et al. 2005, 2006). Several recent X-ray observations, however, might be more easily interpreted if the Herbig AeBe stars themselves were magnetically active (e.g., Telleschi et al. 2007; Günther \& Schmitt 2009; Huenemoerder et al. 2009).

Until the present observations, EC 95 was believed to be a single intermediate-mass young star, and was, therefore, not expected to show magnetic activity. Indeed, Smith et al. (1999) and Giardino et al. (2007) did propose that the nonthermal radio emission as well as the X-ray emission from that source might be provided by a low-mass companion rather than by the AeBe protostar. Preibisch (1999) also mentioned that a low-mass companion could be at the origin of the X-ray emission, though he initially did not consider this possibility particularly likely since the X-ray luminosity of EC 95 based on ROSAT observations appeared to be nearly three orders of magnitude larger than that of typical T Tauri stars. However, more recent XMM-Newton observations have shown that the Xray luminosity of EC 95 is significantly smaller than originally thought and is in fact within the range of normal $\mathrm{T}$ Tauri stars (albeit near the top of the X-ray luminosity function). Thus, it appears plausible again that the X-ray emission might come from a low-mass companion.

Our observations have revealed that EC 95 is a binary system most likely composed of an $\sim 4-5 M_{\odot}$ primary and a lowmass T Tauri companion. Moreover, the low-mass companion (EC 95b) appears to be usually brighter at radio wavelengths than the more massive primary (Table 1; Figure 3). Given the existing correlation between the X-ray and the radio emission of $\mathrm{T}$ Tauri stars, one would expect EC $95 \mathrm{~b}$ to also be the brightest member of the system at X-ray wavelengths. Thus, the magnetic activity from EC 95 is likely to be largely dominated by a lowmass companion rather than by the intermediate mass primary, as proposed by Smith et al. (1999). However, our observations show that the intermediate-mass star EC 95a, although on average somewhat weaker than EC 95b, is also a non-thermal radio emitter. ${ }^{7}$ Thus, the existence of a low-mass companion does not entirely solve the mystery of magnetic activity in the intermediate-mass system EC 95. A mechanism capable of generating magnetic activity on the $4-5 M_{\odot}$ star EC 95a still has to be identified.

Two classes of processes have been proposed to explain intrinsic magnetic activity in intermediate-mass stars. The first one invokes wind or accretion shocks (see Skinner \& Yamauchi 1996 for a detailed discussion). For reasonable values of the wind velocity and of the free-fall speed $\left(\sim 500 \mathrm{~km} \mathrm{~s}^{-1}\right)$, these processes generate plasma temperatures $(k T)$ below $1 \mathrm{keV}$. If a single temperature is assumed, the best fit to the $\mathrm{X}$-ray spectrum of EC 95 is obtained for $k T=2.8 \mathrm{keV}$ (Preibisch 2003a), a value too high to be reconciled with the idea of a shock origin. However, a slightly better fit to the spectrum is obtained for a two-temperature plasma model with $k T_{1}=0.7 \mathrm{keV}$ and $k T_{2}=$ $3.0 \mathrm{keV}$. Could the high-temperature plasma trace the T Tauri star, and the low-temperature component a shock associated with the intermediate-mass star? In this scenario, the variability of the radio emission from EC 95a would be ascribed to changes

\footnotetext{
7 One could argue that the magnetic activity might come from yet another low-mass companion of EC 95a, on a very tight orbit. We consider this possibility highly unlikely because its presence would generate a wobble in the measured positions of EC 95a and the relative position of EC 95b. We find no such wobbling at the level of about 0.2 mas, so the semimajor axis of the companion would have to be less than about $0.1 \mathrm{AU}$, or $20 R_{\odot}$.
}

in the accretion/ejection activity of the star, so fairly large variations in that activity would be required. During episodes of enhanced activity, the X-ray and radio emissions would be expected to rise, so the relative contribution of the lowtemperature component would be expected to increase. Thus, a comparison between X-ray observations obtained during a low and a high state of EC 95a would provide a valuable test of that possibility.

A second mechanism capable of producing magnetic activity in an intermediate-mass star is related to the existence of a thin temporary convective layer on the surface of the star. Two mechanisms that might lead to the appearance of such a layer are substellar deuterium burning during the very early stages of stellar evolution (Palla \& Stahler 1993) and differential rotation in a rapidly rotating star (Tout \& Pringle 1995; Skinner et al. 2004). For a 3-4 $M_{\odot}$ star, Palla \& Stahler (1993) estimated that deuterium burning could last for about $10^{5} \mathrm{yr}$. A corona powered by rotation, on the other hand, could survive for up to about $10^{6} \mathrm{yr}$. Since the age of EC 95 is of the order of $10^{5} \mathrm{yr}$, both mechanisms could in principle maintain a corona over the required timescale, although a rotation-driven corona would accommodate the age of EC 95a somewhat more comfortably. Independently of the mechanism creating it, a thin convective layer would power a magnetic activity similar to that occurring in T Tauri stars. Thus, the flare-like variability observed in our radio observations of EC 95a (Figure 3(a)) would be naturally interpreted in this scenario. We note, finally, that EC 95a is a fairly fast rotator ( $V \sin i=56 \pm 1 \mathrm{~km} \mathrm{~s}^{-1}$; Doppmann et al. 2005) as would be required for a temporary convective layer to be powered by differential rotation. We conclude that all the known properties of EC 95a would be readily interpreted if its magnetic activity were due to a rotation-driven convective layer, and we favor this mechanism over alternative ones.

\subsection{Luminosities of EC 95}

In the previous section, we have argued that although EC 95a is also magnetically active, the radio and X-ray emissions from EC 95 are dominated by the contribution of EC 95b, which we identify with a T Tauri star. We now examine whether or not the relation between the $\mathrm{X}$-ray, the radio, and the bolometric luminosities of EC 95b is consistent with that possibility.

Recent surveys (e.g., Güdel et al. 2007) have shown that the $\mathrm{X}$-ray luminosity of T Tauri stars can vary from about $10^{28}$ to about $10^{31} \mathrm{erg} \mathrm{s}^{-1}$. Moreover, X-ray luminosities in excess of $10^{32} \mathrm{erg} \mathrm{s}^{-1}$ have been observed during flares (e.g., Preibisch $2003 \mathrm{~b}$ ). Scaled to the distance found here, the X-ray luminosity ${ }^{8}$ of EC 95 appears to be about $7 \times 10^{31} \mathrm{erg} \mathrm{s}^{-1}$. This, of course, includes both sources in the system. If our interpretation is correct, however, most of that luminosity is to be ascribed to EC 95b, which would appear to be somewhat brighter in X-rays than typical quiescent T Tauri stars. Its X-ray luminosity would be more comparable to that of T Tauri stars during flares.

The typical X-ray to bolometric luminosity ratio for $\mathrm{T}$ Tauri stars is $5 \times 10^{-4}$, but it can be as high as a few times $10^{-3}$ (e.g., Preibisch 2003a). Using the total bolometric luminosity of EC 95, we obtain $L_{X} / L_{\text {bol }} \sim 1.6 \times 10^{-4}$, near the low end of typical values found in T Tauri stars. However, if the X-ray

\footnotetext{
8 Here, we scale the value of the luminosity obtained by Preibisch (2003a) using a single-temperature fit. A two-temperature model yields a slightly better fit and a somewhat higher luminosity. However, the temperature of the second component in that fit $(k T \sim 0.7 \mathrm{keV})$ is quite atypical of T Tauri stars. If that second component is real, it is likely associated with a different kind of structure, such as an accretion shock (see Section 4.2).
} 
luminosity is dominated by the low-mass T Tauri star EC 95b, one should calculate the ratio using the bolometric luminosity of EC 95b only. Since the total bolometric luminosity of EC 95 is largely dominated by the intermediate mass primary, the ratio estimated above must be severely underestimated. For instance, if EC $95 \mathrm{~b}$ were a $5 L_{\odot}$ T Tauri star, then $L_{X} / L_{\text {bol }}$ for that star would be $3.5 \times 10^{-3}$, near the upper end of the range for $\mathrm{T}$ Tauri stars.

The mean $3.6 \mathrm{~cm}$ flux of EC $95 \mathrm{~b}$ in the first seven epochs of our observations is $1.8 \mathrm{mJy}\left(\equiv 1.8 \times 10^{-26} \mathrm{erg} \mathrm{s}^{-1} \mathrm{~cm}^{-2} \mathrm{~Hz}^{-1}\right)$. This corresponds to a radio luminosity $L_{R}=3.8 \times 10^{17} \mathrm{erg} \mathrm{s}^{-1} \mathrm{~Hz}^{-1}$. Thus, the $\mathrm{X}$-ray to radio luminosity ratio for EC $95 \mathrm{~b}$ is $L_{X} / L_{R} \sim 1.84 \times 10^{14} \mathrm{~Hz}\left(\equiv 10^{14.3} \mathrm{~Hz}\right)$. This ratio for stellar coronae is typically $L_{X} / L_{R} \sim 10^{15.5} \mathrm{~Hz}$ with a dispersion of about 1 dex (Güdel 2004; Benz \& Güdel 1994). Thus, in terms of its radio to X-ray luminosity ratio, EC 95b appears to be within the normal range for stellar coronae.

In summary, if EC 95b is indeed at the origin of most of the magnetic activity in the EC 95 system, then it would have to be interpreted as a fairly X-ray bright $\mathrm{T}$ Tauri star (with an X-ray luminosity more typical of a flaring than of a quiescent star) but with otherwise normal global properties (in terms of $L_{X} / L_{\text {bol }}$ or $L_{X} / L_{R}$ ). We have shown earlier that EC 95 is a tight binary system. Close binarity is known to enhance magnetic activity in stars-for instance, through tidal synchronism, which tends to increase the angular velocity of the members of binary systems. Thus, it is plausible that EC 95b is (because of its binarity) a particularly magnetically active, but otherwise normal, $T$ Tauri star.

\subsection{A Solution to the Extinction Mystery of EC 95?}

There is an unsolved mystery regarding the extinction to EC 95. While the reddening deduced from near infrared photometric observations seems to imply a very large absorption $\left(A_{V}=36\right.$; Preibisch 1999), the extinction obtained from the $\mathrm{X}$-ray spectrum is significantly smaller-albeit still very large $\left(A_{V} \sim 17\right)$. Several mechanisms have been proposed to resolve this conundrum (see the discussion by Preibisch 2003a) but no fully satisfactory explanation has yet been provided. The fact that two sources are detected in EC 95 might naturally explain this mystery. The infrared luminosity is clearly largely dominated by the more massive star EC 95a. Thus, the extinction deduced from infrared observations $\left(A_{V} \sim 36\right)$ is the extinction to that specific star. As discussed earlier, the X-ray emission, on the other hand, is likely to be dominated by the low-mass companion EC 95b, so the extinction deduced from X-ray data $\left(A_{V} \sim 17\right)$ refers to that second star (see, indeed, Giardino et al. 2007). The different values obtained from infrared and X-ray observations, therefore, suggest that the extinction to EC 95a is about 20 mag larger than that to EC 95b. Since the two sources are separated by only about 15 mas ( $\equiv 7 \mathrm{AU})$, the structure responsible for the excess of emission toward EC 95a must be very compact-for instance in the form of a dense circumstellar disk.

Interestingly, such a structure is known to exist in at least one other Herbig AeBe candidate: T Tauri Sa. Based on observations of rovibrational lines of CO, Duchêne et al. (2005) have shown that T Tau Sa is surrounded by a compact structure which would generate an extinction $A_{V}$ of about 90 mag if the dust-to-gas ratio in that structure were similar to that of the interstellar medium. They interpret that structure as a nearly edge-on, compact $(R \sim$ $2-3 R_{\odot}$ ) circumstellar disk. It is noteworthy that EC 95 a and $\mathrm{T}$ Tau Sa are similar in several respects. Both are most likely young Herbig $\mathrm{AeBe}$ stars orbited by a low-mass companion (T Tau Sb and EC 95b, respectively) located at about $10 \mathrm{AU}$. Thus, in both cases, the circumstellar disks are expected to be tidally truncated to about $2-3 \mathrm{AU}$. The rovibrational $\mathrm{CO}$ lines in EC 95 are somewhat fainter than those in $\mathrm{T} \mathrm{Tau} \mathrm{Sa,}$ and the $K$-band veiling of EC $95\left(r_{K} \sim 0.1\right.$; Doppmann et al. $2005)$ is also smaller than that of T Tau Sa $\left(r_{K} \sim 2\right.$; Duchêne et al. 2003). Thus, the amount of circumstellar material around EC 95a must be significantly smaller than that around T Tau Sa. Still, a scaled-down version of the compact disk surrounded T Tau Sa, consistent with the fainter CO lines and smaller veiling of EC 95, might well provide the 20 mag of extinction required to reconcile the infrared and X-ray extinction determinations.

\subsection{Stellar Density of SVS 4}

We mentioned in Section 1 that SVS 4 is believed to be one of the densest sub-clusters in the Milky Way. Given the determination of the distance to SVS 4 given here, it is useful to re-examine this issue. Eiroa \& Casali (1989) identified 11 young stellar objects in an angular diameter of $\sim 50^{\prime \prime}$. Assuming a typical mass of $1 M_{\odot}$ for each object, they obtained stellar mass densities between $4 \times 10^{3}$ and $10^{5} M_{\odot} \mathrm{pc}^{-3}$ (the broad range reflected the uncertainty on the distance to Serpens). The 11 objects in SVS 4 are now known to be embedded in a common faint nebulosity covering an area of $\sim 35^{\prime \prime} \times 35^{\prime \prime}$ (Eiroa et al. 2008). This implies a diameter of $\sim 0.07 \mathrm{pc}$ for the SVS 4 group. The mass of EC 95 is about $5 M_{\odot}$, so if we assume the other 10 objects in SVS 4 to be $1 M_{\odot}$ stars, we obtain a total mass of $15 M_{\odot}$ for the sub-cluster. This yields a stellar mass density of about $8 \times 10^{4} M_{\odot} \mathrm{pc}^{-3}$. This is consistent with the estimates of Eiroa \& Casali (1989) and confirms that SVS 4 is one of the densest concentrations of pre-main-sequence stars known in the Galaxy.

\section{THE DISTANCE TO THE SERPENS CORE AND CLOUD}

As we mentioned earlier, the distance to Serpens has been a matter of controversy during the past several decades. In Table 2, we compile the distance estimates obtained by different authors over the years. All these estimates are indirect, and most of them rely on spectroscopic parallaxes either to estimate the distance directly to Serpens members, or to measure extinction as a function of distance for objects in the direction of Serpens. Interestingly, several of those studies are based on the same samples of stars (or at least on strongly overlapping samples), but reach widely different conclusions. This is the consequence of two main sources of uncertainty. The first one is the assignment of a spectral type to the stars considered, which sets their absolute magnitudes. The second is the determination of the extinction factor $R_{V}$, which is required to translate reddening into extinction. There has been some discussion about the appropriate value of $R_{V}$ for Serpens (e.g., de Lara et al. 1991), and the different assumptions made by different authors are at least partly responsible for the wide range of distances reported in the literature.

In a recent review, Eiroa et al. (2008) argue that the most recent distance estimates seem to converge toward a value of $230 \pm 20 \mathrm{pc}$ for the Serpens molecular cloud. The case for such a convergence, however, is not immediately apparent from Table 2. In fact, that claim appears to be largely based on the results presented by Straižys et al. (2003). Yet, these authors do not calculate the distance to the Serpens cloud, but the distance to the Aquila Rift, which is a large system of dark clouds, that 
Table 2

Measured Distances to the Serpens Molecular Cloud

\begin{tabular}{|c|c|c|c|c|c|}
\hline Authors & Year & Method & $\begin{array}{c}\text { Distance } \\
(\mathrm{pc})\end{array}$ & $\begin{array}{c}\sigma_{d} \\
(\mathrm{pc})\end{array}$ & Sub-region \\
\hline Racine & 1968 & Distance modulus to HD170634 (optical) & 440 & & S68 \\
\hline Strom et al. & 1974 & Distance modulus to HD170634 (infrared) & 440 & & S68 \\
\hline Chavarria-K et al. & 1988 & Spectroscopy, $u v b y \beta$ and $J H K L M$ photometry to four stars & 245 & 30 & Serpens cloud \\
\hline Zhang et al. & 1988 & Distance modulus to three stars & 650 & 180 & Serpens core \\
\hline & & Kinematic distance of $\mathrm{CO}$ and $\mathrm{NH}_{3}$ lines & 600 & & \\
\hline de Lara \& Chavarria & 1989 & Infrared photometry to seven stars & 296 & 34 & Serpens cloud \\
\hline de Lara et al. & 1991 & Extinction measurements of five stars & 311 & 38 & Serpens cloud \\
\hline Chiar & 1997 & Infrared photometry to seven stars & 425 & 45 & Serpens cloud \\
\hline Straižys et al. & 1996 & Vilnius photometry of 105 stars & 259 & 37 & Serpens cloud \\
\hline
\end{tabular}

includes Serpens but is significantly more extended. ${ }^{9}$ Moreover, Straižys et al. (2003) only claim to have measured the distance to the front edge of the Aquila Rift, which they place at $225 \pm$ $55 \mathrm{pc}$. They further argue that the depth of the complex might be about $80 \mathrm{pc}$, so depending on the position of Serpens within the complex, its distance might be somewhat larger. Thus, from the results of Straižys et al. (2003), 230 pc appears as a lower limit to the distance to Serpens rather than as the most probable value. In a previous paper, Straižys et al. (1996) did estimate the distance specifically to the Serpens cloud; they obtained $259 \pm 37$ pc. Their more recent Aquila Rift paper includes 80 of the stars used in that 1996 paper, as well as 400 other stars distributed over the rest of the Aquila Rift region. Thus, it does not provide (nor claim to provide) a new and independent estimate of the distance to Serpens, but rather-as mentioned above-a measurement of the distance to the front edge of the Aquila Rift.

The distance obtained here is based on a trigonometric parallax measurement, and, therefore, does not rely on any assumption regarding the nature of the star or the properties of the interstellar medium in the Serpens and Aquila Rift clouds. There is no question that EC 95 is located at $414.9_{-4.3}^{+4.4} \mathrm{pc}$. On the other hand, we only measured the distance to that one star, whereas previous works based on indirect determinations (such as those of Straižys et al. 1996) relied on much larger samples. One must, therefore, address the issue of the relevance of the distance measured here for the entire Serpens complex. In this respect, it is important to point out that EC 95 is not just any star located in the direction of Serpens. As we have seen earlier, it is a very young Herbig AeBe star. Such intermediate-mass stars are not born in isolation, but as part of small clusters. Since EC 95 is one of at least 11 pre-main-sequence stars in the compact SVS 4 region, there is no doubt that it is physically associated with that small cluster (see also Eiroa \& Casali 1989) and that the distance measured here is also the distance to SVS 4. In addition, SVS 4 is universally acknowledged to be part of the Serpens core region (e.g., Eiroa \& Casali 1992), and its members (including EC 95) have always been considered part of the Serpens young stellar cluster (Eiroa \& Casali 1992). Thus, we argue that the distance to EC 95 measured here also provides an accurate estimate of the distance to the Serpens core. We note further that the Serpens core is only about $5^{\prime}$ across on the plane of the sky ( $0.6 \mathrm{pc}$ at a distance of $415 \mathrm{pc})$. Thus, depth effects are not expected to add significantly to the error budget on the determination of the distance to the core. As a consequence, we

\footnotetext{
9 In total, the Aquila Rift covers about $400 \mathrm{deg}^{2}$ of the sky, whereas Serpens is only about $6 \mathrm{deg}^{2}$.
}

argue that the distance to the Serpens core should henceforth be assumed to be $415 \pm 5 \mathrm{pc}$.

With an angular diameter of about $3^{\circ}$, the Serpens cloud is much larger than the Serpens core. At a distance of $415 \mathrm{pc}$, this would correspond to a physical diameter of about $20 \mathrm{pc}$. The depth of the Serpens complex is also likely to be of that order, and depending on the location of the core relative to the rest of the complex, the mean distance appropriate for the Serpens cloud might be up to $20 \mathrm{pc}$ less or $20 \mathrm{pc}$ more than the distance to the core. Thus, we argue that a conservative estimate of the mean distance to the Serpens cloud would be $415 \pm 25 \mathrm{pc}$.

This value is significantly larger than the figure reported by Straižys et al. (1996) for the Serpens molecular cloud $(d=259 \pm$ $37 \mathrm{pc}$ ). Since the total extent of the Serpens cloud on the plane of the sky is only about $20 \mathrm{pc}$, it is extremely unlikely to be $150 \mathrm{pc}$ deep. Instead, we argue that several unrelated regions are likely to coexist along the line of sight. As mentioned above, Serpens is one of the many dust clouds forming the large structure known as the Aquila Rift. Straižys et al. (2003) have shown that the front edge of that structure is at about $230 \mathrm{pc}$ and that its depth is about $80 \mathrm{pc}$. The discrepancy between our result and that of Straižys et al. (1996) could be readily explained if the Serpens molecular cloud were not physically associated with the Aquila Rift, but instead located behind it. In this situation, since the method used by Straižys et al. (1996) is sensitive to the position of the first obscuring material located along the line of sight, it would have naturally picked out the foreground Aquila Rift clouds rather than the more distant Serpens region. Indeed, in their concluding remarks, Straižys et al. (2003) state that "both W40 and the Serpens molecular cloud are seen projected on the very dark foreground created by the dust lanes of the Aquila Rift." Such a superposition along the same line of sight of different clouds at different distances is not entirely surprising since that line of sight passes only slightly above the Galactic plane, but it certainly warrants caution when considering individual regions in that direction. For instance, it is quite plausible that some of the patchy structures seen in the direction of Serpens on optical plates are in fact associated with foreground material in the Aquila Rift.

\section{CONCLUSIONS AND PERSPECTIVES}

In this paper, we have presented multi-epoch VLBA observations of EC 95, a young stellar object located in the SVS 4 sub-cluster of the Serpens molecular core. Our data demonstrate that EC 95 is in fact a tight binary system with a separation of about 15 mas. The primary (EC 95a) appears to be a 4-5 $M_{\odot}$ intermediate-mass Herbig AeBe protostar, whereas the secondary (EC 95b) is most likely a low-mass T Tauri. At radio wavelengths, the secondary is on average brighter than the 
primary. It is, therefore, also likely to dominate the fairly bright $\mathrm{X}$-ray emission from the system. The primary, on the other hand, contributes most of the infrared and the bolometric luminosity of EC 95. This might naturally explain why the extinction to EC 95 based on infrared observations was systematically found to be much larger than the extinction based on X-ray data.

Interestingly, both members of EC 95 appear to be nonthermal radio emitters. While a low-mass T Tauri star such as EC $95 \mathrm{~b}$ is expected to generate that type of emission, an intermediate-mass such as EC 95a is not. We discussed several mechanisms that could explain the presence of non-thermal emission on EC 95a, and argue that the observed properties of EC 95a might be most readily explained if it possessed a corona powered by a thin, rotation-driven convective layer.

The trigonometric parallax of EC 95 appears to be $\pi=$ $2.41 \pm 0.02$ mas, corresponding to a distance of $414.9_{-4.3}^{+4.4} \mathrm{pc}$. We argue that this implies a distance to the Serpens core of $415 \pm 5 \mathrm{pc}$ and to the Serpens molecular cloud of $415 \pm 25 \mathrm{pc}$. This is significantly larger than previous distance estimates based on measurements of the extinction suffered by stars in the direction of Serpens $(d \sim 260 \mathrm{pc})$. A possible explanation for this discrepancy is that these measurements correspond to the distance to the clouds associated with the Aquila Rift located in the foreground of the Serpens cloud.

Since our observations resolve EC 95 into a binary, future radio monitoring of that system will allow us to measure the dynamical mass of that system. To our knowledge, this will be the first time that a dynamical mass is obtained for a young Herbig AeBe system, and it will enable us to uniquely constrain theoretical pre-main-sequence evolutionary for intermediatemass stars. The orbital period of the system appears to be 10-20 years, so observations in the next two decades will be required to obtain an accurate mass estimate.

The Serpens cloud is about $20 \mathrm{pc}$ across on the plane of the sky, so it is likely to be also about $20 \mathrm{pc}$ deep. Observations similar to those presented here for a sample of young stars distributed across the cloud would help determine the structure of the region and would allow us to further constrain the mean distance to this important region of star formation.

S.D., L.L., and L.F.R. acknowledge the financial support of DGAPA, UNAM, and CONACyT, Mexico. R.M.T. acknowledges support by the Deutsche Forschungsgemeinschaft (DFG) through the Emmy Noether Research grant VL 61/3-1. NRAO is a facility of the National Science Foundation operated under cooperative agreement by Associated Universities, Inc.

\section{REFERENCES}

Benz, A. O., \& Güdel, M. 1994, A\&A, 285, 621

Berghöfer, T. W., Schmitt, J. H. M. M., Danner, R., \& Cassinelli, J. P. 1997, A\&A, 322, 167

Chavarria-K, C., de Lara, E., Finkenzeller, U., Mendoza, E. E., \& Ocegueda, J. 1988, A\&A, 197, 151

Chiar, J. E. 1997, PhD thesis, Rensselaer Polytechnic Institute

Condon, J. J. 1997, PASP, 109, 166

de Lara, E., \& Chavarria, C. K. 1989, RevMexAA, 18, 180

de Lara, E., Chavarria-K., C., \& Lopez-Molina, G. 1991, A\&A, 243, 139

Doppmann, G. W., Greene, T. P., Covey, K. R., \& Lada, C. J. 2005, AJ, 130, 1145

Duchêne, G., Ghez, A. M., McCabe, C., \& Weinberger, A. J. 2003, ApJ, 592, 288

Duchêne, G., Ghez, A. M., McCabe, C., \& Ceccarelli, C. 2005, ApJ, 628, 832
Eiroa, C. 1992, in ESO Scientific Report, Low Mass Star Formation in Southern Molecular Clouds, ed. B. Reipurth (Garching: ESO), 197

Eiroa, C., \& Casali, M. M. 1989, A\&A, 223, L17

Eiroa, C., \& Casali, M. M. 1992, A\&A, 262, 468

Eiroa, C., Djupvik, A. A., \& Casali, M. M. 2008, in Handbook of Star Forming Regions, Vol. II: The Southern Sky, ASP Monograph Publications, Vol. 5, ed. B. Reipurth (San Francisco, CA: ASP), 693

Eiroa, C., Torrelles, J. M., Curiel, S., \& Djupvik, A. A. 2005, AJ, 130, 643

Feigelson, E. D., \& Montmerle, T. 1999, ARA\&A, 37, 363

Forbrich, J., Massi, M., Ros, E., Brunthaler, A., \& Menten, K. M. 2007, A\&A, 469,985

Giardino, G., Favata, F., Micela, G., Sciortino, S., \& Winston, E. 2007, A\&A, 463,275

Greisen, E. W. 2003, Astrophys. Space Sci. Libr., 285, 109

Güdel, M. 2004, A\&ARv, 12, 71

Güdel, M., et al. 2007, A\&A, 468, 353

Günther, H. M., \& Schmitt, J. H. M. M. 2009, A\&A, 494, 1041

Haisch, K. E., Jr., Barsony, M., Greene, T. P., \& Ressler, M. E. 2002, AJ, 124, 2841

Harvey, P., Merín, B., Huard, T. L., Rebull, L. M., Chapman, N., Evans II, N. J., \& Myers, P. C. 2007, ApJ, 663, 1149

Hubrig, S., et al. 2009, A\&A, 502, 283

Huenemoerder, D. P., Schulz, N. S., Testa, P., Kesich, A., \& Canizares, C. R. 2009, ApJ, 707, 942

Loinard, L., Mioduszewski, A. J., Rodríguez, L. F., González, R. A., Rodríguez, M. I., \& Torres, R. M. 2005, ApJ, 619, L179

Loinard, L., Torres, R. M., Mioduszewski, A. J., \& Rodríguez, L. F. 2008, ApJ, 675, L29

Loinard, L., Torres, R. M., Mioduszewski, A. J., Rodríguez, L. F., GonzálezLópezlira, R. A., Lachaume, R., Vázquez, V., \& González, E. 2007, ApJ, 671,546

Menten, K. M., Reid, M. J., Forbrich, J., \& Brunthaler, A. 2007, A\&A, 474, 515

Mioduszewski, A. J., \& Kogan, L. 2009, AIPS Memo 110, Strategy for Removing Tropospheric and Clock Errors using DELZN

Montmerle, T., Wade, G., Landstreet, J., Mènard, F., Grosso, N., \& Feigelson, E. D. 2005, in Protostars and Planets V, ed. B. Reipurth, D. Jewitt, \& K. Keil (Tucson, AZ: Univ. Arizona Press), 8112

Palla, F., \& Stahler, S. W. 1993, ApJ, 418, 414

Parker, E. N. 1955, ApJ, 122, 293

Pittard, J. M., \& Dougherty, S. M. 2006, MNRAS, 372, 801

Pontoppidan, K. M., van Dishoeck, E. F., \& Dartois, E. 2004, A\&A, 426, 925

Preibisch, T. 1998, A\&A, 338, L25

Preibisch, T. 1999, A\&A, 345, 583

Preibisch, T. 2003a, A\&A, 410, 951

Preibisch, T. 2003b, A\&A, 401, 543

Racine, R. 1968, AJ, 73, 233

Reid, M. J., \& Brunthaler, A. 2004, ApJ, 616, 872

Rodríguez, L. F., Moran, J. M., Ho, P. T. P., \& Gottlieb, E. W. 1980, ApJ, 235, 845

Skinner, S. L., Güdel, M., Audard, M., \& Smith, K. 2004, ApJ, 614, 221

Skinner, S. L., \& Yamauchi, S. 1996, ApJ, 471, 987

Smith, K., Güdel, M., \& Benz, A. O. 1999, A\&A, 349, 475

Stelzer, B., Flaccomio, E., Montmerle, T., Micela, G., Sciortino, S., Favata, F., Preibisch, T., \& Feigelson, E. D. 2005, ApJS, 160, 557

Stelzer, B., Micela, G., Hamaguchi, K., \& Schmitt, J. H. M. M. 2006, A\&A, 457, 223

Straižys, V., Černis, K., \& Bartašiūtë, S. 1996, Balt. Astron., 5, 125

Straižys, V., Černis, K., \& Bartašiūtë, S. 2003, A\&A, 405, 585

Strom, S. E., Grasdalen, G. L., \& Strom, K. M. 1974, ApJ, 191, 111

Strom, S. E., Vrba, F. J., \& Strom, K. M. 1976, AJ, 81, 638

Telleschi, A., Güdel, M., Briggs, K. R., Skinner, S. L., Audard, M., \& Franciosini, E. 2007, A\&A, 468, 541

Torres, R. M., Loinard, L., Mioduszewski, A. J., \& Rodríguez, L. F. 2007, ApJ, 671,1813

Torres, R. M., Loinard, L., Mioduszewski, A. J., \& Rodríguez, L. F. 2009, ApJ, 698,242

Tout, C. A., \& Pringle, J. E. 1995, MNRAS, 272, 528

Wade, G. A., Alecian, E., Grunhut, J., Catala, C., Bagnulo, S., Folsom, C. P., \& Landstreet, J. D. 2009, arXiv:0901.0347

Winston, E., et al. 2007, ApJ, 669, 493

Xu, Y., Reid, M. J., Zheng, X. W., \& Menten, K. M. 2006, Science, 311, 54

Zhang, C. Y., Laureijs, R. J., Wesselius, P. R., \& Clark, F. O. 1988, A\&A, 199, 170 